\title{
Dissociation Dynamics of Phenetole Cations by Photoelectron Photoion Coincidence
}

\author{
John Riley and Tomas Baer \\ Department of Chemistry, University of North Carolina, Chapel Hill, North Carolina, USA
}

The dissociation rates of phenetole ions have been measured as a function of the ion internal energy by the method of photoelectron photoion coincidence spectrometry. The loss of ethylene to produce the phenol ion is the only dissociation pathway from its onset at $9.17 \mathrm{eV}$ up to at least $12 \mathrm{eV}$. An activation energy of $1.64 \pm 0.06 \mathrm{eV}$ with an assumed activation entropy of $+1.8 \pm 4 \mathrm{cal} / \mathrm{mol}-\mathrm{K}$ is derived from fitting the statistical theory decay rates to the measured rates. The transition state energy lies $\sim 1 \mathrm{eV}$ below the thermochemical dissociation limit to the $\mathrm{C}_{2} \mathrm{H}_{5}^{+}+\mathrm{C}_{6} \mathrm{H}_{5} \mathrm{O} \cdot$ products. It is thus unlikely that an ion-radical complex is involved in the production of the phenol ion. (J Am Soc Mass Spectrom (1991, 2, 464-469)

I ons of the alkyl phenyl ethers dissociate at low energies by losing an alkene and producing the phenol ion. This reaction is associated with the well-known metastable alkyl phenyl ether ions. Among the molecules that follow this pattern are ethyl phenyl ether (phenetole), propyl phenyl ether, and the various butyl phenyl ethers. Although this reaction has been studied by a variety of groups [1-5], the mechanism for this reaction is still under debate [3-5].

A reverse activation energy for this reaction has been postulated because the measured appearance energy for the product phenol ion and the corresponding alkene is considerably higher than the difference in the thermochemical energies of these species and the ground state alkyl phenyl ether. Such a barrier is consistent with a rearrangement reaction. However, it is well known that appearance energies provide only upper limits to true barrier height. This is because of the kinetic shift which is the additional ion energy required to raise the dissociation rate so that products are formed on the time scale of the experiment.

A method for determining the true barrier height involves measuring the dissociation rates of the ion as a function of the ion internal energy and extrapolating these rates with the aid of the statistical theory of unimolecular decay (RRKM/QET) to the dissociation onset. Such an analysis was performed on the phenetole ion by Lemaire et al. [2] by multiphoton ionization (MPI) in a three-color laser experiment. Two laser colors were used to ionize the phenetole molecule

Address reprint request to Tomas Baer, Department of Chemistry, University of North Carolina, Chapel Hill, NC 27599-3290. while a third laser photodissociated the ion. The measured rates as a function of the ion internal energy were fit to the statistical theory. The extrapolated onset energy for dissociation was reported as $1.46 \mathrm{eV}$, which is lower than the reported appearance energies which ranged from 1.9 to $2.6 \mathrm{eV}$ [4]. The difference between these two values was attributed to the kinetic shift. However, the measured rates could not be reconciled with the rates predicted by the statistical theory over the whole energy range investigated. In addition, Lemaire et al. [2] observed the production of $\mathrm{C}_{5} \mathrm{H}_{6}^{+}$, which is a well-known product from the dissociation of phenol [6], but is not energetically accessible via a simple photodissociation of the phenetole ion.

Some questions remain concerning the transition state structure for ethylene loss from phenetole. Bouchoux [1] analyzed the metastable peak in terms of a "tight" transition state in which a hydrogen atom is transferred from the alkyl group to the oxygen atom. In contrast, Chronister and Morton [3] have suggested that isopropyl phenyl ether (and probably all of the alkyl phenyl ethers) react via a lonsely bound phenoxy radical complex, $\left[\mathrm{R}^{+}-\mathrm{OPh} \cdot\right]$, which presumably would account for both the $\mathrm{H}$ atom transfer step as well as the $H / D$ isotopic scrambling observed at higher energies. However, no scrambling is observed at low energies [5]. The transferred $\mathrm{H}$ atom comes exclusively from the terminal methyl group in phenetole. In addition, if the activation energy is as low as claimed by Lemaire et al. [2], a complex leading to the production of $\mathrm{R}^{+}$ions may lie at too high an energy.

In view of these problems, we have undertaken the investigation of the phenetole ion dissociation rates using the method of photoelectron photoion coincidence (PEPICO) [7]. 


\section{Experimental}

The PEPICO technique has been described extensively in the literature [7]. Briefly, vacuum ultraviolet radiation from a many-line hydrogen discharge, grating dispersed in a $0.5-\mathrm{m}$ monochromator, ionized the gas-phase sample in a uniform $10 \mathrm{~V} / \mathrm{cm}$ electric field acceleration zone. Initially, zero kinetic energy photoelectrons were selected by passing them through a steradiancy energy analyzer [8]. The ions were accelerated in a uniform 3-cm electric field before entering a $26-\mathrm{cm}$ drift region. The electrons and ions provided the start and stop signals for a time to amplitude converter, whose output was sent to a multichannel analyzer. The resulting ion time-of-flight (TOF) distributions were accumulated for $3-5 \mathrm{~h}$.

The ions are detected in coincidence with zero energy electrons, so the ion internal energy is given by:

$$
E=h v-I P+E_{t h}
$$

where $h v$ is the photon energy, IP is the ionization potential, and $E_{t h}$ is the thermal energy of the neutral molecule. The total energy resolution was determined to be $105 \mathrm{meV}$ by measuring the full width at half maximum of the first vibrational band in the photoelectron spectrum of acetylene.

The methods for accounting for the initial thermal energy of the neutral molecules have been shown to depend on how close the total ion energy is to the threshold for fragmentation $[9,10]$. For energies near the dissociation onset, a sum of reaction rates must be used, each rate in the sum being the true microcanonical rate at each energy in the thermal distribution. On the other hand, for energies sufficiently above the onset, the thermal energy can be included as an average [6]. Owing to the kinetic shift in our experiments, the data presented represent fragmentations at energies far enough above the onset to include thermal effects as an average. Therefore, a total vibrational and rotational internal energy of $0.20 \mathrm{eV}$ was added to all photon energies to obtain the correct experimental ion internal energy.

The phenetole sample was obtained from Aldrich Chemical [Milwaukee, WI $(99.9 \%)$ ]. We noticed a considerable phenol impurity ( $m / z$ 94 signals at energies well below published dissociation onsets) and verified this by gas chromatography. Removal of the impurity proved difficult. Because data acquisition times were hours per experiment, it is believed that the phenetole decomposed in the PEPICO inlet system.

\section{Results and Discussion}

\section{Reaction Rates}

The only fragment ion observed in the present photodissociation study of phenetole ions was $\mathrm{C}_{6} \mathrm{H}_{6} \mathrm{O}^{+}$ $\cdot(m / z 94)$, resulting from the loss of $\mathrm{C}_{2} \mathrm{H}_{4}$. The experimental TOF distributions for the $\mathrm{m} / \mathrm{z} 94$ ion at several photon energies are shown in Figure 1. The peak shapes are asymmetric because of the metastable parent ions that dissociate in the acceleration region. This asymmetry decreases as the ion internal energy increases because of the increasing reaction rate. The asymmetry is less apparent in these data than in previous studies of other ions because a symmetric peak centered at $40.5 \mu \mathrm{s}$, resulting from the previously mentioned phenol impurity, is superimposed on the $m / z 94$ fragment peak. This impurity signal was present well below the onset for $\mathrm{C}_{2} \mathrm{H}_{4}$ loss from phenetole, and disappeared at photon energies below the phenol ionization energy of $8.47 \mathrm{eV}$ [11]. The solid lines in Figure 1 are the calculated ion TOF distributions assuming the indicated unimolecular dissociation rates. The phenol ion signal at $40.5 \mu \mathrm{s}$ was accounted for by adding a symmetric peak to the asymmetric TOF simulation. The implicit assumption in this treatment of the data is that all of the energyselected phenetole ions decay by the slow rate. We thus ignore the possibility that the dissociation rate is two-component. This is a reasonable assumption because of the large activation energy and because Lemaire et al. [2] did not report any evidence of two-component decay rates.

The experimentally derived rates are plotted as a function of energy in Figure 2. The two energy scales, photon energy and ion internal energy (including initial thermal energy), differ by the amount $E_{t h}-I P$. The error bars for the experimental points were determined from the minimum and maximum rates that gave acceptable agreement between calculated and experimental TOF distributions.

The data of Lemaire et al. [2] are shown as the shaded region in Figure 2. Clearly, the PEPICO-determined reaction rates are about an order of magni-

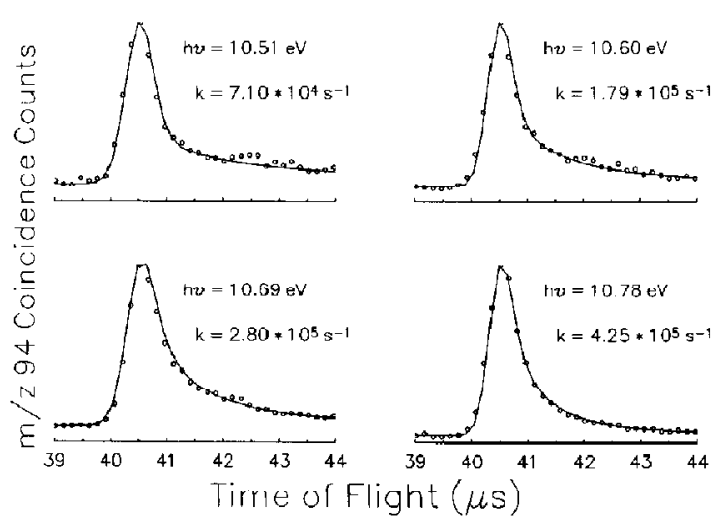

Figure 1. Fragment ion $\left(\mathrm{C}_{6} \mathrm{H}_{5} \mathrm{OH}^{+}\right)$TOF distributions from ionizing phenetole at the indicated photon energies. The points are the experiment results; the solid lines are calculated assuming the indicated dissociation rates. The major part of the symmetric peak at $40.5 \mu \mathrm{s}$ is a result of photoionizing phenol molecules which were inevitably produced through decomposition of phenetole in the gas inlet system. 
tude smaller than those derived from the three-color laser experiment. In addition to the lower rates, the energy dependence of the PEPICO-derived rates have a larger slope.

\section{RRKM/QET Calculations of Dissociation Rates}

The onset for $\mathrm{C}_{2} \mathrm{H}_{4}$ loss has been determined by monoenergetic electron impact studies [4] to be 10.30 $\mathrm{eV}$ and from the metastable signal appearance energy [4] to be $10.5 \mathrm{eV}$. These values agree with our findings. That is, the lowest energy at which useful data for the metastable dissociation could be determined was $10.49 \mathrm{eV}$ (see Figure 1). Below that energy only the symmetric phenol ion peak was observed. The measured rate of $7 \times 10^{4} \mathrm{~s}^{-1}$ (which is close to the minimum rate measurable in our instrument) at 10.49 $\mathrm{eV}$ indicates that this onset is determined by the rate of dissociation near the threshold rather than by the true activation barrier, $E_{0}$. To determine the "true" barrier, the experimental rate versus the energy curve was fit with calculated curves from the RRKM rate equation:

$$
k(E)=\frac{\sigma \int_{0}^{E-E_{0}} N^{\#}(\epsilon) d \epsilon}{h N(E)}=\frac{\sigma G^{\#}\left(E-E_{0}\right)}{h N(E)}
$$

where $\sigma$ is the reaction path degeneracy, $L$ is the total ion internal energy, $h$ is Planck's constant, $N$ and $N^{\#}$ are the densities of states for the molecular ion and transition state, respectively, and $G^{*}$ is the sum of states given by the integral in eq 1 . The input parameters for the calculation are $E_{0}$ and the molecular ion and transition state vibrational frequencies. $A$ steepest decent algorithm was used to evaluate the state densities [12].

A first attempt at fitting the rate data was carried out with the vibrational frequencies (Table 1) obtained

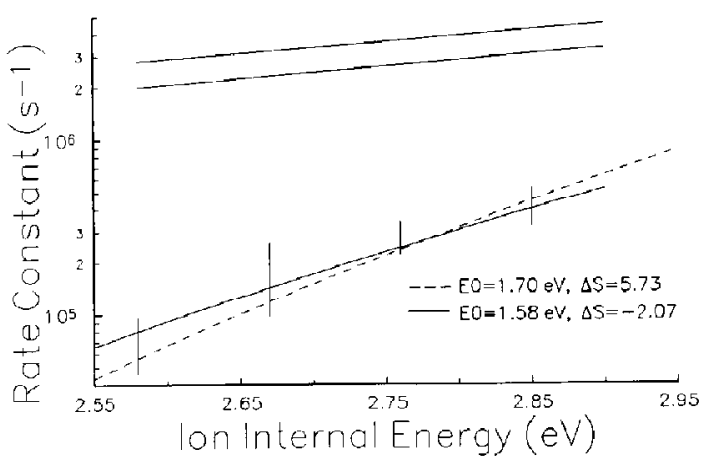

Figure 2. The dissociation rate as a function of the ion internal energy. The vertical lines are the PEPICO experimental results. The shaded region is taken from the work of Lemaire et al. [2]. The solid and dashed lines through the PEPICO data are from RRKM/QET calculations for two different transition states. The different transition state vibrational frequencies lead to the indicated activation energies $\left(E_{0}\right)$ and entropies of activation. from Green [13] for neutral phenetole (which we assumed to be valid for the phenetole molecular ion) and from Bouchoux [1] for the transition state. Bouchoux reported two feasible sets of transition state frequencies. We chose the set corresponding to the enol (phenol) product rather than the keto (cyclohexadienone) product because the $\mathrm{C}_{6} \mathrm{H}_{6} \mathrm{O}^{+}$product from alkyl phenyl ethers has been shown to be a phenolic structure by collisional activation studies [14, 15]. The results of deuteration studies $[3-5,16]$ indicate that, at energies near threshold, the methyl protons are primarily involved in the transfer, but at higher energies the methylenic protons also rearrange. These labeling results are consistent with the mechanism for rearrangement proposed by Bouchoux [1] and the vibrational frequencies used for our calculations.

The only parameter adjusted in these calculations was the barrier height, $E_{0}$. The fit from this calculation is shown as the solid curve in Figure 2, which corresponds to $E_{0}=1.58 \mathrm{eV}$. It is evident that the scatter in the data allow for other transition state frequencies. The slope of the calculated curve can be made steeper by "loosening" the transition state, that is, by assuming lower transition state vibrational frequencies. An RRKM calculation with the transition state vibrational frequencies multiplied by 0.90 also gave a good fit (dashed line in Figure 2) when the activation energy was assumed to be $1.70 \mathrm{eV}$. If we consider the two calculated $k(E)$ curves to be the two limiting fits, we obtain an average activation energy of $1.64 \pm 0.06 \mathrm{eV}$. The difference between this $E_{0}$ and our appearance energy ( $2.56 \mathrm{eV}$ internal energy) gives a kinetic shift for this reaction of $0.92 \mathrm{eV}$. That is, phenctolc cations require $-0.9 \mathrm{eV}$ of energy in excess of the activation energy to dissociate rapidly enough for the observation of fragment ions in our experiment.

The ratio of transition state frequencies to molecular ion frequencies is related to the entropy of activatiun, $\Delta S^{\#}$. For reactions having a positive $\Delta S^{\#}$, the transition state is termed "loose" and these reactions do not typically have reverse barriers (defined to be $\left.E_{0}-\Delta H_{r x n}^{0}\right)$. In contrast, reactions with reverse barriers, such as rearrangement reactions, are characterized by negative entropies of activation. The Bouchoux frequencies in Table 1 yield an activation entropy of $-2.1 \mathrm{cal} / \mathrm{mol}-\mathrm{K}$ at $1000 \mathrm{~K}$. The negative value is thus consistent with the rearrangement assumed for the loss of ethylene from phenetole. On the other hand, the "loose" transition state with the lower frequencies gave a $\Delta S^{*}$ of $+5.7 \mathrm{cal} / \mathrm{m}-\mathrm{K}$. Because of the limited range of experimental rates illustrated in Figure 2, we are unable to determine which of the two models is better.

Lemaire et al. [2] also reported RRKM/QET calculated rates which passed through their data (shaded region in Figure 2) but with a considerably higher slope. The frequencies used by these authors are similar to ours except that an internal hindered rota- 
Table 1. Phenetole vibrational frequencies

\begin{tabular}{rrrrrrrrrrr}
\hline \multicolumn{10}{l}{ Phenetole molecule (and molecular ion) ${ }^{\mathrm{a}} \mathrm{cm}^{-1}$} \\
\hline \hline 3096 & 3096 & 3068 & 3068 & 3040 & 2985 & 2985 & 2985 & 2884 & 2884 \\
1603 & 1592 & 1498 & 1460 & 1460 & 1460 & 1448 & 1390 & 1335 & 1292 \\
1246 & 1246 & 1246 & 1175 & 1155 & 1117 & 1085 & 1080 & 1047 & 1026 \\
998 & 975 & 953 & 922 & 901 & 829 & 797 & 755 & 755 & 690 \\
614 & 509 & 427 & 408 & 347 & 298 & 238 & 180 & 195 & 58 \\
44 & & & & & & & & & \\
\hline \hline Transition state ("tight" $)^{\mathrm{b}}, \mathrm{cm}^{-1}$ & & & & & & & \\
\hline \hline 3096 & 3096 & 3068 & 3068 & 3040 & 2985 & 2985 & 2985 & 2050 & 2884 \\
1603 & 1592 & 1498 & 1460 & 1460 & 1000 & 1448 & 1390 & 1335 & 1292 \\
1246 & 1246 & 1246 & 1175 & 1155 & $\# \#$ & 1085 & 1080 & 1047 & 1026 \\
998 & 975 & 953 & 1270 & 901 & 829 & 797 & 756 & 765 & 690 \\
614 & 509 & 427 & 408 & 347 & 212 & 238 & 180 & 500 & 58 \\
44 & & & & & & & & & \\
\hline
\end{tabular}

\footnotetext{
Taken from Green [13].

"Frequencies for "tight" transition state taken from Bouchoux I1]. Frequencies in bold ara different from molecular ion frequencies. The assumed reaction coordinate is indicated by the symbol \#. The "Ionse" transition state frequancies were obtained by multiplying the Bouchoux frequencies by 0.90 .
}

tion frequency of $58 \mathrm{~cm}^{-1}$ was converted to $200 \mathrm{~cm}^{-1}$. In addition, they used an activation energy of $1.46 \mathrm{eV}$. With these parameters we in fact obtain calculated rates that are only a factor of two higher than our calculated solid line and with a slope that is slightly less than our slope. That is, our calculated rates using the Lemaire parameters gave rates that are an order of magnitude lower than those reported by Lemaire. We do not understand the origin of this discrepancy.

\section{Energetics}

The heats of formation at $298 \mathrm{~K}$ and $0 \mathrm{~K}$ for several species important in the formation of phenol from phenetole cations are shown in Table 2 and the $0 \mathrm{~K}$ data are shown diagrammatically in Figure 3 . The dissociation onset of phenetole to phenol cations, determined from the RRKM calculations to be 9.77

Table 2. Heats of formation of relevant molecules and ions

\begin{tabular}{|c|c|c|}
\hline Molecule/ion & $\Delta H_{r 298}^{0}(\mathrm{~kJ} / \mathrm{mol})$ & $\Delta H_{f 0}^{0}(\mathrm{~kJ} / \mathrm{mol})$ \\
\hline$\overline{\mathrm{C}_{6} \mathrm{H}_{5} \mathrm{OC}_{2} \overline{\mathrm{H}_{5}}}$ & $-101.7^{\circ}$ & $-72.98^{\mathrm{b}}$ \\
\hline $\mathrm{C}_{6} \mathrm{H}_{5} \mathrm{OC}_{2} \mathrm{H}_{5}^{+}$ & $682.4^{\circ}$ & $711.2^{b}$ \\
\hline $\mathrm{C}_{5} \mathrm{H}_{5} \mathrm{OH}^{+}$ & $722^{d}$ & $733^{d}$ \\
\hline $\mathrm{C}_{2} \mathrm{H}_{4}$ & $52.3^{\circ}$ & $60.7^{6}$ \\
\hline $\mathrm{C}_{5} \mathrm{H}_{6}^{+}$ & $960^{4}$ & $970^{\circ}$ \\
\hline $\mathrm{CH}_{3} \mathrm{CHCO}$ & $-104,6^{x}$ & $-95.0^{7}$ \\
\hline $\mathrm{CO}$ & $-113.8^{9}$ & $-110.5^{\mathrm{e}}$ \\
\hline $\mathrm{C}_{2} \mathrm{H}_{5}^{+}$ & $918^{a}$ & $914^{a}$ \\
\hline $\mathrm{C}_{6} \mathrm{H}_{5} \mathrm{O}$ & $47.7^{a}$ & $64.2^{\mathrm{g}}$ \\
\hline
\end{tabular}

"Lias et al. [11].

Calculated by using the vibrational frequencies from Green [13].

${ }^{c}$ Determined from the reported ionization potential of $8.13 \mathrm{eV}$ by Lemaire et al. [2]:

dFraser-Monteiro et al. [6].

"Wagman et al. [17].

${ }^{4}$ Calculated (estimated) by using vibrational frequencies of propanal from Shimanouchl [18].

${ }^{0}$ Estimated by comparison with phenoxy ion in ref 11 .
$\mathrm{eV}$, is also shown in Figure 3. Collisionally induced dissociation (CID) study of the product ions has shown conclusively that the ionic product has the phenol ion structure [4]. The only reasonable structure for the $\mathrm{C}_{2} \mathrm{H}_{4}$ neutral fragment is ethylene. Because the combined heats of formation at $0 \mathrm{~K}$ of the phenol cation and ethylene is $8.99 \mathrm{eV}$, we conclude therefore that this reaction proceeds with a reverse activation barrier of $0.78 \mathrm{eV}$. Such a reverse barrier is generally characterized by substantial kinetic energy release (KER) to the neutral and ionic fragments. The KERs were not determined in the present study, but an average KER of $65 \mathrm{meV}$ has been derived from the $T_{0.5}$ value of 30 $\mathrm{meV}$ measured in a mass-analyzed ion kinetic energy spectrometry (MIKES) study $[4,5]$.

The statistically expected KER can be calculated by using the Klots [19] formulation, which is based on a statistical distribution of energy among all of the product degrees of freedom. The internal energy of the metastable ions in the spectrometer of Blanchette

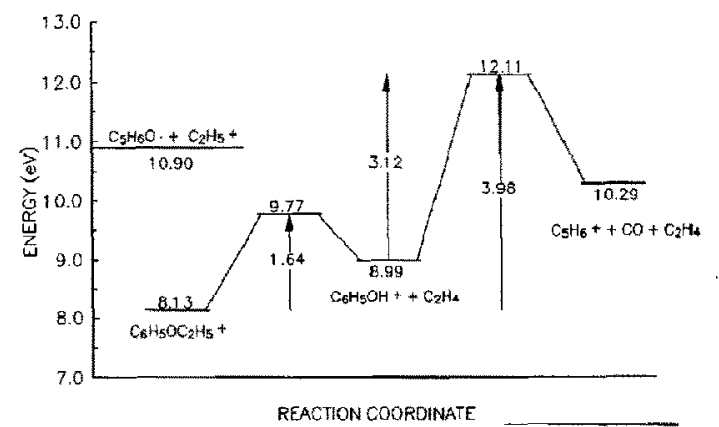

Figure 3. The $0 \mathrm{~K}$ energy diagram for the phenetole ion disso ciation to phenol ions and ultimately to cyclopentadiene. The dissociation limit to the ethyl ion and the phenoxy radical is also shown. 
et al. [4] can be estimated from our $k(E)$ curve in Figure 2. If we assume that the metastable ions dissociate in the second field-free region at times ranging between 6 and $15 \mu \mathrm{s}$, we estimate that the dissociating parent ions have a mean internal energy of $2.5 \pm 0.2$ $\mathrm{eV}$, thereby leaving $1.76 \mathrm{eV}$ excess energy for the products. The Klots value for the average KER at this total ion energy is $0.085 \mathrm{eV}$. The good agreement of the statistically expected and measured KER indicates that significant energy redistribution takes place among the reaction products after passing through the transition state region.

\section{The Reaction Mechanism}

What do these results tell us about the reaction mechanism? Chronister and Morton [3] suggested for the case of propyl phenyl ether that the reaction passes through an ion radical complex, $\mathrm{R}^{+}-\mathrm{PhO}$. . It was proposed that the interaction at long distances mediates the $H$ atom transfer step which leads to the phenol ion product. The separation between these two units was assumed to be between 7 and $15 \AA$ and the potential energy of the complex at these distances was calculated to be -21 and $-2.6 \mathrm{~kJ} / \mathrm{mol}$ relative to the $\mathrm{R}^{+}+\mathrm{PhO}$ - products [3]. If we apply these internuclear distances to the phenetole ion, we predict that this complex should lie at an energy of 245 and 263 $\mathrm{kJ} / \mathrm{mol}(2.54$ and $2.73 \mathrm{eV})$ relative to the ground state energy of the phenetole ion. This energy is signifcantly above the derived transition state energies for either the tight $(1.58 \mathrm{eV})$ or the loose $(1.70 \mathrm{eV})$ transition states. We conclude, therefore, that the ion-neutral complex mechanism involving a long-range interaction between $\mathrm{R}^{+}$and $\mathrm{PhO}$ - is not consistent with the phenetole ion energelics derived from our rate measurements. On the other hand, the simple $\mathrm{H}$ atom transfer step via a tight four-membered ring transition state as proposed by Bouchoux [1] is consistent with the rate measurements.

The nearly statistical kinetic energy release is rather remarkable considering the large reverse activation barrier. The calculation of KER for reactions with a reverse activation barrier is a complicated matter. The important parameter is the shape of the potential energy surface in the exit part of the reaction. This multidimensional surface is difficult to calculate and the partitioning of the available energy even more so. Christie et al. [20] approached the problem by comparing the calculated geometry and the vector motions of the atoms in the transition state with the structure of the dissociation products. Although the agreement between this approach and the experimental results is not perfect, it does provide a qualitative insight into the problem. Thus, we can infer that, if the transition state "looks like" the products, or if the motion in the transition state leads directly to products, the available energy will be channeled principally into translational energy. However, if the transition state structure is significantly different from the products, the products have a chance to redistribute the available energy among all the degrees of freedom.

A tight, four-center transition state in which the $\mathrm{H}$ atom is transferred from the terminal methyl group to the oxygen certainly does not "look like" the products. That is, considerable rearrangement of the products must take place after passing through the transition state, thereby promoting energy redistribution. It is interesting that Harnish and Holmes [5] have some results that suggest that a considerable amount of internal energy remains in the product ethylene. According to our statistical theory calculation, the product energies are distributed as follows: $.085 \mathrm{eV}$ translational energy, $0.21 \mathrm{eV}$ rotational energy, $1.17 \mathrm{eV}$ phenol ion vibrational energy, and $0.29 \mathrm{eV}$ ethylene vibrational energy. It is not clear whether this $0.29 \mathrm{eV}$ internal energy in the ethylene fragment is sufficient to account for the different CID behavior observed by Harnish and Holmes [5]. It may well be that the total vibrational energy is statistical, but that the partitioning of this energy between the ethylene and the phenol ion products is not statistical.

\section{Other Reaction Products}

In addition to the $m / z 94$ fragment ion, Lemaire et al. [2] also observed an $m / z 66\left(\mathrm{C}_{5} \mathrm{H}_{6}^{+}\right)$fragment. The appearance energy of $11 \mathrm{eV}$ for this ion corresponds to an ion internal energy of $-2.8 \mathrm{eV}$. $\Lambda$ s previously mentioned, no fragment ions other than $m / z 94$ were observed at energies up to $3.44 \mathrm{eV}$ in the PEPICO experiments. The observation of the $m / z 66$ fragment ion, which most likely has the cyclopentadiene struc ture, is not readily explained. Although the phenol ion itself dissociates to $\mathrm{C}_{-} \mathrm{C}_{5} \mathrm{II}_{6}^{+}$via loss of $\mathrm{CO}$, it does so with rates in excess of $10^{6} \mathrm{~s}^{-1}$ only at ion internal energies greater than $4.4 \mathrm{eV}$ [5]. Thus, the sequential reaction of phenetole to phenol to cyclopentadiene ions could only be observable at energies in excess of $13.4 \mathrm{eV}$. As a result, Lemaire et al. [2] proposed that c- $\mathrm{C}_{5} \mathrm{H}_{6}^{+}$is produced directly from phenetule ions along with a $\mathrm{C}_{3} \mathrm{H}_{4} \mathrm{O}$ neutral fragment. To explain the fact that the $m / z 94$ and 66 ions were produced with rates that differ by a factor of 5, Lemaire et al. [2] further proposed that the latter is produced from an excited electronic state, or from a different isomer of the phenetole radical cation.

The thermochemical dissociation limit for the pro-

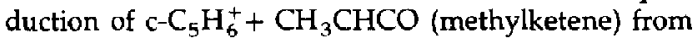
phenetole is $9.83 \mathrm{eV}$, which is well below the claimed onset of $11 \mathrm{eV}$ by Lemaire et al. [2]. Thus, this reaction cannot be ruled out on the basis of the thermochemistry. However, the fact that this product is not observed in our one-photon ionization suggests that the laser experiment is producing phenetole ions in states that are not produced in the one-photon ionization. Although the laser production of phenetole ions in different electronic states, or even in different isomeric forms, cannot be ruled out, a more likely pussi- 
bility is that an additional photon is absorbed by the phenetole ion, which would bring the total energy above the dissociation limit of the product phenol ion. Such sequential photon absorptions have been observed in the photodissociation of butadiene ions even at low laser powers, where it was nearly impossible to prevent the absorption of the second photon [21]. It is also possible that a fraction of the phenetole molecules produced in the intermediate $S_{1}$ state by the first laser may dissociate. These dissociation products may then absorb further photons to finally yield the $\mathrm{C}_{5} \mathrm{H}_{6}^{+}$ product ion.

\section{Conclusion}

The formation of phenol cations from metastable phenetole ions was investigated in the energy range $2.56-2.88 \mathrm{eV}$. The only product observed up to an ion energy of $3.44 \mathrm{eV}$ is the loss of ethylene. By fitting the experimental rate versus energy data with RRKM rate curves, we found the barrier to dissociation to be $1.64 \pm 0.06 \mathrm{eV}$. The derived reverse activation energy of $0.78 \mathrm{eV}$ is consistent with a tight transition state. However, the $\Delta S^{\#}$ of $+1.8 \pm 4 \mathrm{cal} / \mathrm{m}-\mathrm{K}$ contains sufficiently large error bars to prevent us from drawing any mechanistic conclusions from it. On the other hand, the rather small release of kinetic energy (as measured by MIKES) leads us to conclude that considerable rearrangement of atoms takes place after the reaction passes through the transition state.

Finally, the transition state energy of $1.64 \mathrm{eV}$ is found to lie significantly below the proposed ion-neutral complex, which has an energy of -2.65 $\pm 0.1 \mathrm{eV}$. This $1 \mathrm{-eV}$ mismatch in energies is a strong indication that the ion-neutral complex does not play a role in the dissociation of phenetole ions.

\section{Acknowledgments}

We are grateful for discussions with Joel Lemaire and Illiana Dimicoli concerning the discrepancies between the PEPICO and the laser-based results. We thank John Holmes for discussions and for making available to us the preprint of ref 5 . We acknowledge the U.S. Department of Energy for the support of this work.

\section{References}

1. Bouchoux, G. Org. Mass Spectrom. 1978, 13, 184.

2. Lemaire, J.; Dimicoli, I.; Botter, R. Chem. Phys. 1987, 115, 129.

3. Chronister, E. L.; Morton, T. H. J. Am. Chem. Soc. 1990, $112,133$.

4. Blanchette, M. C.; Holmes, J. L.; Lossing, F. P. Org. Mass Spectrom. 1989, 24, 673.

5. Harnish, D.; Holmes, J. L. J. Am. Chem. Soc. 1991, in press.

6. Fraser-Monteiro, M. L.; Fraser-Monteiro, L.; deWit, U.; Baer, T. I. Phys. Chem. 1984, 88, 3622.

7. Baer, T. Adv. Chem. Phys. 1986, 64, 111.

8. Baer, T.; Peatman, W. B.; Schlag, E. W. Chem. Phys. Lett. 1969, 4, 243.

9. Brand, W. A.; Baer, T. Int. I. Mass Spectrom. Ion Processes 1983, 49, 103.

10. Baer, T.; Kury, R. Chem. Phys. Lett. 1983, 92, 659.

11. Lias, S. G.; Bartmess, J. E.; Lievman, J. F.; Holmes, J. L.; Levin, R. D.; Mallard, W. G. J. Phys. Chem. Ref. Data 1988, 17,Suppl. 1.

12. Forst, W. Theory of Unimolecular Reactions, Academic: New York, 1973.

13. Green, J. H. S. Spectrochim. Acta 1962, 18, 39.

14. Borchers, F.; Levsen, K.; Beckey, H. D. Int. J. Mass Spectrom. Ion Processes 1976, 21, 125.

15. Maquestiau, A.; Van Havervbeke, Y.; Flammang, R.; De Meyer, C.; Das, K. G.; Reddy, G. S. Org. Mass Spectrom. 1977, 12, 631 .

16. Benoit, F. M.; Harrison, A. G. Org. Mass Spectrom. 1976, 11, 599.

17. Wagman, D. D.; Evans, W. H.; Barker, V. B.; Schum, R. H.; Halow, I.; Bailey, S. M.; Churney, K. L.; Nuttall, R. L. J. Phys. Chem. Ref. Data 1982, 11, Suppl. 2.

18. Shimanouchi, T. Natl. Stand. Ref. Data Ser. Natl. Bur. Stand. 1972, 39.

19. Klots, C. E. J. Chem. Phys. 1973, 58, 5364.

20. Christie, J. R,; Derrick, P. J.; Richard, G. J. J. Chem. Soc. Farad. Trans. II 1978, 74, 304.

21. Bunn, T. L.; Baer, T. J. Chem. Phys. 1986, 85, 636. 